\title{
Promoter methylation attenuates SHP1 expression and function in patients with primary central nervous system lymphoma
}

\author{
JING LIU ${ }^{1}$, YAMING WANG ${ }^{2}$, XUEFEI SUN ${ }^{1}$, NAN JI $^{3}$, SHENGJUN SUN $^{4}$, YAJIE WANG $^{5}$, \\ FUSHENG LIU ${ }^{6}$, QU CUI ${ }^{1}, \mathrm{CHEN} \mathrm{WANG}^{7}$ and YUANBO LIU ${ }^{1,7}$ \\ ${ }^{1}$ Department of Hematology, Beijing Tiantan Hospital, Capital Medical University; ${ }^{2}$ Department of Neurosurgery, \\ Navy General Hospital; ${ }^{3}$ Department of Neurosurgery, Institute of Neurological Sciences, Beijing Tiantan Hospital, \\ Capital Medical University; ${ }^{4}$ Neuroimaging Center, Beijing Tiantan Hospital, Capital Medical University; \\ ${ }^{5}$ Core Laboratory for Clinical Medical Research, Beijing Tiantan Hospital, Capital Medical University; \\ ${ }^{6}$ Institute of Neurological Sciences, Beijing Tiantan Hospital, Capital Medical University; ${ }^{7}$ Department of \\ Internal Medicine, Beijing Tiantan Hospital, Capital Medical University, Beijing 100050, P.R. China
}

Received June 26, 2016; Accepted August 9, 2016

DOI: $10.3892 /$ or.2016.5308

\begin{abstract}
The Src homology region 2 domain-containing phosphatase-1 (SHP1) is a critical negative regulator involved in the JAK/STAT signaling pathway. The SHP1 gene has been proposed as a candidate tumor suppressor in solid and hematological malignancies and promoter methylation is an important biological process in controlling tumorigenesis. However, the detailed roles of SHP1 promoter methylation in the pathogenesis of primary central nervous system lymphoma (PCNSL) is largely unknown. In the present study, we evaluated the correlation between SHP1 expression and promoter methylation in patients with PCNSL. Thirty-three patients with PCNSL were included. We evaluated SHP1 protein expression levels by immunohistochemistry and the SHP1 promoter methylation profile by pyrosequencing analysis. For cases $(n=8)$ with a good yield of total protein, SHP1 phosphorylation (pSHP1) and STAT3 protein expression levels were further analyzed by western blot analysis to uncover the molecular impact of SHP1 promoter methylation on downstream signaling pathways. In this study, a lower expression of SHP1 protein level was observed in $16 / 33$ cases $(48.5 \%)$ of PCNSL. SHP1 promoter
\end{abstract}

Correspondence to: Professor Yuanbo Liu, Department of Hematology, Beijing Tiantan Hospital, Capital Medical University, 2F, South Ward Building, 6 Tiantan Xili, Dongcheng, Beijing 100050, P.R. China

E-mail: yuanbol@ccmu.edu.cn

Professor Chen Wang, Department of Internal Medicine, Beijing Tiantan Hospital, Capital Medical University, 6 Tiantan Xili, Dongcheng, Beijing 100050, P.R. China

E-mail: wangchen-tr2002@163.com

Key words: primary central nervous system lymphoma, SHP1, methylation, phosphorylation, STAT3 methylation was predominant in $29 / 33$ cases $(87.9 \%$ ) with a mean methylation level of $31.7 \pm 36.5 \%$. The mean methylation level of the SHP1 promoter was significantly elevated in patients with a lower SHP1 protein expression, compared with those showing a higher SHP1 protein expression $(50.3 \pm 38.9$ vs. $14.2 \pm 24.0 \%, p=0.004)$. Further analysis showed that SHP1 protein expression was significantly decreased in patients with a higher SHP1 promoter methylation status $(\mathrm{p}=0.001)$, and such attenuation was correlated with a downregulation of pSHP1 $(\mathrm{p}=0.005)$ and an upregulation of STAT3 protein expression $(\mathrm{p}=0.020)$. Our data demonstrated that epigenetic alterations in the promoter region downregulated SHP1 expression in PCNSL patients. SHP1 promoter methylation was correlated with tyrosine phosphorylation and activation of transcription factor STAT3, which may contribute to the pathogenesis of PCNSL. Therapeutical regimens with epigenetic modifiers may be a potential option for patients with PCNSL.

\section{Introduction}

Primary central nervous system lymphoma (PCNSL) is a distinct type of non-Hodgkin's lymphoma located in the brain, leptomeninges, spinal cord, cerebrospinal fluid (CSF) and intraocular structures, accounting for 2-3\% of all brain tumors and is associated with a dismal prognosis $(1,2)$. Approximately 95\% of PCNSL cases are histologically characterized as diffuse large B-cell lymphoma (DLBCL) (3). Although PCNSL shares some common characteristics with systemic DLBCL, it has unique features based on the transcription profiles and therapeutic protocols (4-8). In clinical practice, patients with PCNSL have greatly benefitted from well-established routine chemotherapy and radiotherapy strategies. However, treating refractory and relapsed PCNSL is still a huge challenge. Novel therapeutical strategies including immunotherapy and targeted therapy by clinically available epigenetic modifiers such as 5-aza-2'-deoxycytidine have been adopted as potential options for these B-cell malignancies, which may shed light on the potential clinical management of PCNSL. 
The development of functional B cells is dependent on the maturation of progenitor cells in the bone marrow, and transcription factor networks play a fundamental role in B cell differentiation. Recent evidence has revealed that most PCNSL cells demonstrate an activated B-cell-like phenotype with constitutive activation of the JAK/STAT and NF-kB signaling pathways $(4,9,10)$. Cytokines including IL-4 and IL-10 were found to stimulate B cell proliferation and survival through upregulation of JAK1 and constitutive activation of STAT3 and STAT6 in PCNSL (11-13). In addition, oncostatin M (OSM) was found to trigger activation of the JAK family, which in turn activated the STAT family in PCNSL, while downregulation of SOCS1 and SOCS3 led to activation of the JAK/STAT signaling pathway $(9,14)$. Molecules involved in the JAK/STAT signaling pathway may be regulating factors contributing to the pathogenesis of PCNSL.

The Src homology region 2 domain-containing phosphatase-1 (SHP1) is an important negative regulator involved in the JAK/STAT signaling pathway through cytokine/growth factors (15). SHP1 is a non-transmembrane protein tyrosine phosphatase predominantly expressed in hematopoietic cells (16-19), participating in various pathways which negatively regulate molecular signals involved in cell activation, proliferation, differentiation, and migration through three categories of receptors (20). These receptors include growth factor receptors with an intrinsic tyrosine kinase activity such as c-kit, EGF, and CSF-1; cytokine receptors such as IL-2R, IL-3R, and Epo-R; and receptor complexes involved in immune response such as TCR and CD5 (21-23). A previous study reported that SHP1 expression in PCNSL reflects the origin of PCNSL from a late germinal center to an early postgerminal center stage (24). It has previously been shown that DLBCL expresses a very low level of SHP1 protein, suggesting that loss of SHP1 may result in the development of DLBCL (25). In hematologic malignancies, the SHP1 gene silenced by aberrant $\mathrm{CpG}$ promoter methylation was found to commonly induce a marked decrease in the SHP1 protein level, indicating that SHP1 expression is predominantly regulated by DNA methylation. Nevertheless, the impact of SHP1 methylation on the development of PCNSL has not been comprehensively characterized.

In this study, we applied immunohistochemistry and pyrosequencing analysis to 33 PCNSL samples to investigate the SHP1 protein expression profile and promoter methylation status. By evaluating molecular alterations in the JAK/STAT signaling pathway through western blot analysis, we aimed to clarify the influence of SHP1 and SHP1 promoter methylation on the pathogenesis of PCNSL.

\section{Patients and methods}

Patients. Clinical data and tumor specimens from 33 immunocompetent patients with PCNSL were analyzed retrospectively. Diagnosis of DLBCL was carried out by histologic review for all specimens according to the Revised European-American Lymphoma and WHO classification (26). For this study, patients were selected on the basis of the availability of paraffin-embedded tumor tissues and fresh-frozen tumor tissues. All patients received chemotherapy regimens based on high-dose methotrexate (HD-MTX). Patients who did not achieve complete remission to HDMTX-based chemotherapy were given rescue whole brain radiotherapy (WBRT). Informed consent from all the patients was obtained. This study was approved by the Beijing Tiantan Hospital Ethics Committee, Capital Medical University.

Tumor specimens. Tumor specimens were obtained during stereotactic biopsy or surgery. Formalin-fixed and paraffin-embedded tumor specimens were used for immunohistochemistry, whereas fresh-frozen tumor specimens were stored at $-80^{\circ} \mathrm{C}$ until extraction of DNA for pyrosequencing analysis. Of the 33 fresh-frozen samples, only 8 samples for protein extraction were used for further western blot analysis due to the quantity of tissue available after pyrosequencing analysis. For normal control samples, we used 11 paraffin-embedded reactive hyperplasia of lymph nodes for immunohistochemistry, peripheral blood from the 33 PCNSL patients for pyrosequencing analysis and 1 fresh-frozen normal lymph node for western blot analysis.

Immunohistochemical analysis. A panel of immunohistochemical staining was performed on $4-\mu \mathrm{m}$ thick sections (EnVision method). Sections were deparaffinized in xylene and dehydrated with ethanol. Antigen retrieval was carried out in a microwave oven for $15 \mathrm{~min}$. And then sections were incubated with a working dilution of each monoclonal antibody: mouse anti-SHP1 monoclonal antibody (Sc-7289, 1:500 dilution; Santa Cruz Biotechnology, Inc., Santa Cruz, CA, USA), monoclonal antibodies against CD10 (ZM-0283, 1:100 dilution), BCL-6 (ZM-0011, 1:50 dilution), MUM1 (ZM-0399; 1:50 dilution) and BCL-2 (ZA-0536, 1:100 dilution) from Beijing Zhongshan Golden Bridge Biotechnology Co., Ltd. (Beijing, China). Reactive hyperplasia of lymph nodes was used as positive controls.

Two pathologists assessed the immunohistochemical staining independently. Fifteen to 20 fields at x400 magnification were analyzed per specimen. SHP1 immunohistochemical staining was evaluated semiquantitatively by estimating the fraction of positive cells according to the cut-off point as published by Sugita et al (24). Tumor tissues with more than $60 \%$ positively stained cells were considered (3+), 30-60\% (2+), 0-30\% (1+), and no staining was considered (-). Only cytoplasmic staining of cells was considered for grading. Staining was considered positive for CD10, BCL-6, and MUM1 when more than $30 \%$ of tumor cells were positively stained (27). For BCL-2, staining was considered positive when more than $50 \%$ of tumor cells expressed the BCL-2 protein according to a previously published method for systemic DLBCL (28).

Pyrosequencing detection. Genomic DNA was extracted from 33 PCNSL frozen tumor tissues and paired peripheral blood by using the QIAamp DNA Mini kit (Qiagen). Pyrosequencing analysis was carried out by Gene-Tech Co., Ltd. (Shanghai, China). For SHP1, 4 CpG sites were studied. PCR primer sequences and sequencing primer are listed as follows: GGTT GTGGTGAGAAATTAATTAGA (forward primer) and CTCC AAACCCAAATAATACTTCA (reverse primer); and GGAG GAGGGAGAGATG (sequencing primer). The annealing temperature was $54^{\circ} \mathrm{C}$. A bisulfitetreated sample $(2 \mu \mathrm{l})$ was amplified in $40 \mu \mathrm{l}$ of reaction 
mixture, containing primers and $2.5 \mathrm{U}$ of Takara Hot Start Taq (Takara Biotechnology Co., Ltd.). The PCR product was purified to obtain single-stranded DNA mixed with $40 \mu \mathrm{l}$ sequencing buffer, containing $0.5 \mu \mathrm{M}$ sequencing primers. Sequencing analysis used the PyroMark ID technique. Methylation data are presented as the percentage of average methylation in all observed $\mathrm{CpG}$ sites. Positive rate using the mean methylation level plus two times the standard deviation (SD) of the control samples as a cut-off point (29).

Western blot analysis. The 8 fresh-frozen tumor tissues and 1 normal lymph node were ground and lysed in lysis buffer and proteinase inhibitor (50:1). After incubation for 30 min on ice, the lysates were subjected to centrifugation at $15,000 \mathrm{rpm}$ for $15 \mathrm{~min}$ at $4^{\circ} \mathrm{C}$. The supernatants were collected after centrifugation. Proteins were quantified using the Bicinchoninic Acid Protein Assay kit (Beyotime, Nantong, China). A total of $40 \mu \mathrm{g}$ protein was loaded onto each lane of $12 \%$ polyacrylamide gel followed by electrotransfer onto polyvinylidene fluoride membranes (Thermo Fisher Scientific, Waltham, MA, USA). The membranes were then blocked with skimmed milk powder in phosphate-buffered saline with $0.05 \%$ Tween-20 (PBST) for $2 \mathrm{~h}$ and incubated with the following primary antibodies at $4{ }^{\circ} \mathrm{C}$ overnight: mouse monoclonal antibody against SHP1 (Sc-7289) was purchased from Santa Cruz Biotechnology, Inc. and used at a dilution of 1:500; rabbit monoclonal antibody against SHP1 phosphorylation (pSHP1) was purchased from Cell Signaling Technology, Inc. (Danvers, MA, USA) and used at a dilution of 1:1,000; mouse monoclonal antibody against STAT3 was purchased from Cell Signaling Technology, Inc. and used at a dilution of 1:1,000; antibody against GADPH was purchased from TransGen Biotech Co., Ltd. (Beijing, China) and used at a dilution of 1:2,000. Membranes were rinsed in PBST three times and incubated with the secondary antibody at a dilution of 1:2,000 (horseradish peroxidase-conjugated monoclonal goat anti-mouse IgG or horseradish peroxidase-conjugated monoclonal goat anti-rabbit IgG; TransGen Biotech Co., Ltd.) at room temperature for $1 \mathrm{~h}$. Membranes were rinsed in PBST three times again and were developed using an enhanced chemiluminescence detection system (GE Healthcare, Piscataway, NJ, USA).

Statistical analysis. Distribution of patient characteristics was applied by the Chi-squared $\left(\chi^{2}\right)$ test. The relationship between SHP1 protein expression and the clinicopathologic variables was evaluated by the Fisher's exact test and $\chi^{2}$ test. Two group comparison was carried out using the Student's t-test or Wilcoxon signed-rank test. All statistical analyses were performed using the SPSS 17.0 software package. A value of $\mathrm{p}<0.05$ was considered statistically significant.

\section{Results}

Patient characteristics. The general information of the PCNSL patients involved in this study is described in Table I. All PCNSLs were proven to be DLBCL by pathological analysis and $29(87.9 \%)$ specimens were obtained from stereotactic biopsies. The median age was 57 years (range, 28-83 years) with a gender ratio of male-to-female of 1.75 . Ten patients $(30.3 \%)$
Table I. Clinical characteristics of the PCNSL patients.

\begin{tabular}{|c|c|}
\hline Characteristics & Patients, n (\% \\
\hline \multicolumn{2}{|l|}{ Age (years) } \\
\hline$>60$ & $12(36.4)$ \\
\hline$\leq 60$ & $21(63.6)$ \\
\hline \multicolumn{2}{|l|}{ Gender } \\
\hline Male & $22(66.7)$ \\
\hline Female & $11(33.3)$ \\
\hline \multicolumn{2}{|l|}{$\mathrm{LDH}$} \\
\hline Elevated & $13(39.4)$ \\
\hline Normal & $20(60.6)$ \\
\hline \multicolumn{2}{|l|}{ Immunophenotype } \\
\hline GCB & $4(12.1)$ \\
\hline Non-GCB & $29(87.9)$ \\
\hline \multicolumn{2}{|l|}{ No. of lesions } \\
\hline 1 & $12(36.4)$ \\
\hline At least 2 & $21(63.6)$ \\
\hline \multicolumn{2}{|l|}{ ECOG performance status } \\
\hline 0 to 1 & $10(30.3)$ \\
\hline At least 2 & $23(69.7)$ \\
\hline \multicolumn{2}{|l|}{ Deep structure involvement } \\
\hline Presence & $24(72.7)$ \\
\hline Absence & $9(27.3)$ \\
\hline Stereotactic biopsy & 29 (87.9) \\
\hline Surgery & $4(12.1)$ \\
\hline \multicolumn{2}{|l|}{ Treatment } \\
\hline HD-MTX based chemotherapy & $33(100)$ \\
\hline \multicolumn{2}{|l|}{ Pathology } \\
\hline DLBCL & $33(100)$ \\
\hline
\end{tabular}

PCNSL, primary central nervous system lymphoma; LDH, lactate dehydrogenase; GCB, germinal center B-cell-like; ECOG, Eastern Cooperative Oncology Group; HD-MTX, high-dose methotrexate; DLBCL, diffuse large B-cell lymphoma.

were scored 0 or 1 by the Eastern Cooperative Oncology Group (ECOG) performance status and the other 23 patients $(69.7 \%)$ scored 2-4 by the same scoring system. Multiple brain lesions were observed in $63.6 \%$ of the patients (21/33) and deep brain structures were compromised in $72.7 \%$ of the patients $(24 / 33)$. Among routine biochemical markers, an elevated serum lactate dehydrogenase (LDH) level was observed in 13 of the 33 patients $(39.4 \%)$. For all samples from these patients, CD10, BCL-6, MUM1 and BCL-2 positive expression was noted in $12.12 \%$ (4/33), $45.46 \%$ (15/33), $87.88 \%$ (29/33) and $78.57 \%$ $(22 / 28)$ of the cases, respectively. According to the Hans classification criteria (30), 4 cases $(12.12 \%)$ were classified as the germinal center B-cell-like (GCB) subgroup, and the other 29 cases $(87.88 \%)$ were non-GCB.

SHP1 protein expression was confirmed by immunohistochemical staining. Immunohistochemical staining was conducted for SHP1 protein expression. Immunoreactivity for 


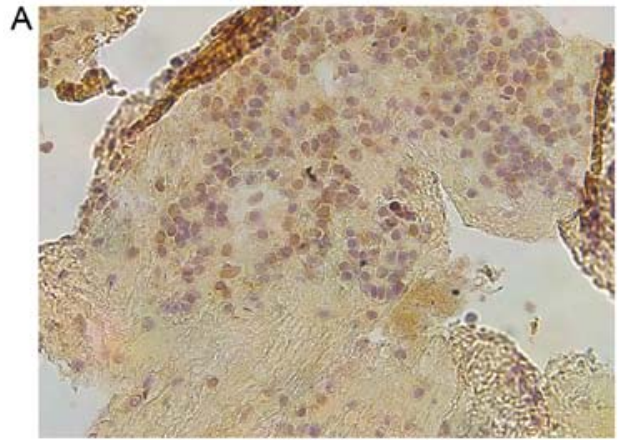

Control, SHP1 (3+), x400

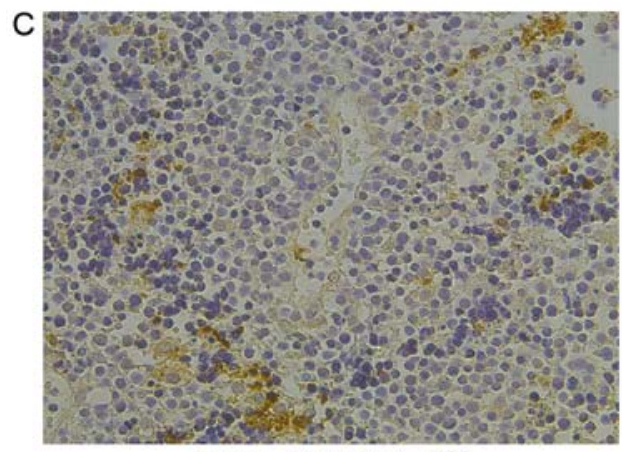

PCNSL, SHP1 (2+), x400

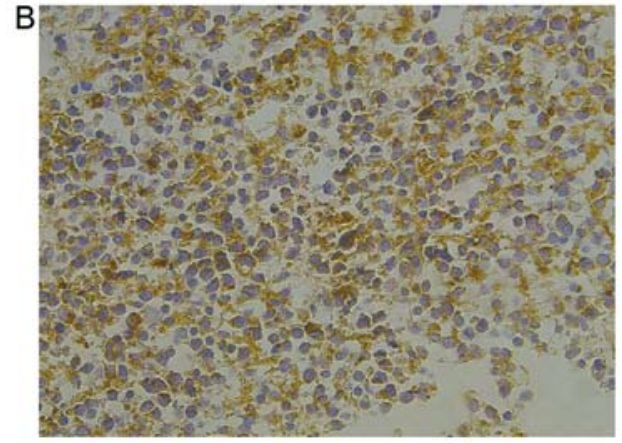

PCNSL, SHP1 (3+), $x 400$

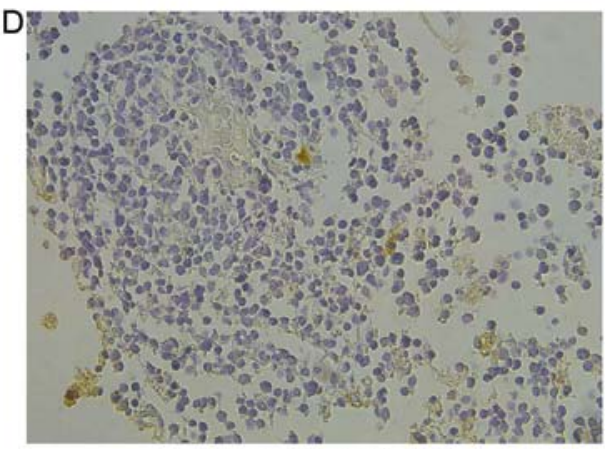

PCNSL, SHP1 (+), $x 400$

Figure 1. SHP1 expression profiles in primary central nervous system lymphoma (PCNSL) and hyperplasia of lymph nodes. (A) Representative high cytoplasmic SHP1 expression (3+) in hyperplasia of lymph nodes. (B) SHP1-positive lymphoma showing cytoplasmic staining (3+). (C) SHP1-positive lymphoma showing cytoplasmic staining (2+). (D) SHP1-positive lymphoma showing cytoplasmic staining (+). SHP1, Src homology region 2 domain-containing phosphatase-1.
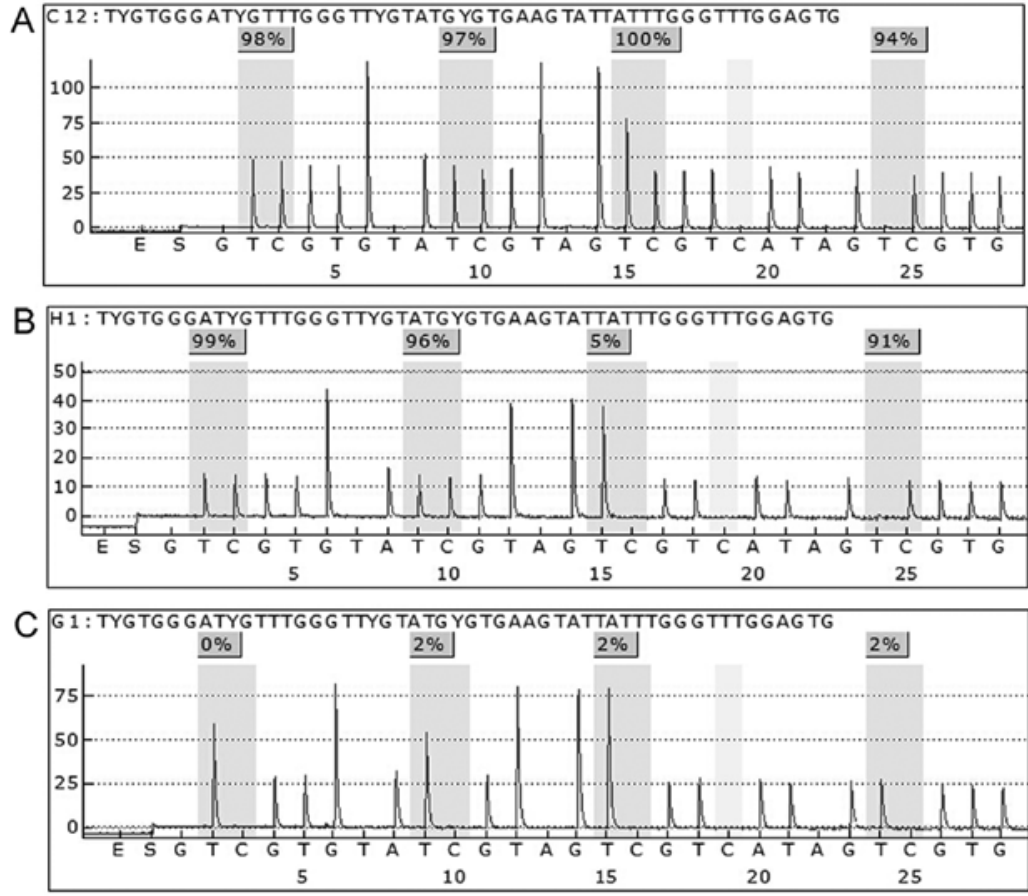

Figure 2. SHP1 promoter methylation status in primary central nervous system lymphoma (PCNSL) by pyrosequencing analysis. (A) Methylation levels ranged from 94 to $100 \%$, with a mean of $97.3 \%$. (B) Methylation levels ranged from 5 to $99 \%$, with a mean of $72.8 \%$. (C) Methylation levels ranged from 0 to $2 \%$, with a mean of $1.5 \%$. The gray highlighted bar marks the tested CpG sites and the percentage readings of cytosine and thymine. SHP1, Src homology region 2 domain-containing phosphatase-1.

SHP1 was strong in reactive hyperplasia of lymph node as a positive control and scored as (3+) (Fig. 1A). SHP1-positive immunostaining was detected in 30 PCNSL tissues $(90.9 \%)$, of which, 17 positive cases showing a high SHP1 expression (over $60 \%$ positive cells) were scored as (3+) (Fig. 1B); while the remaining 13 cases showed a low SHP1 expression with 


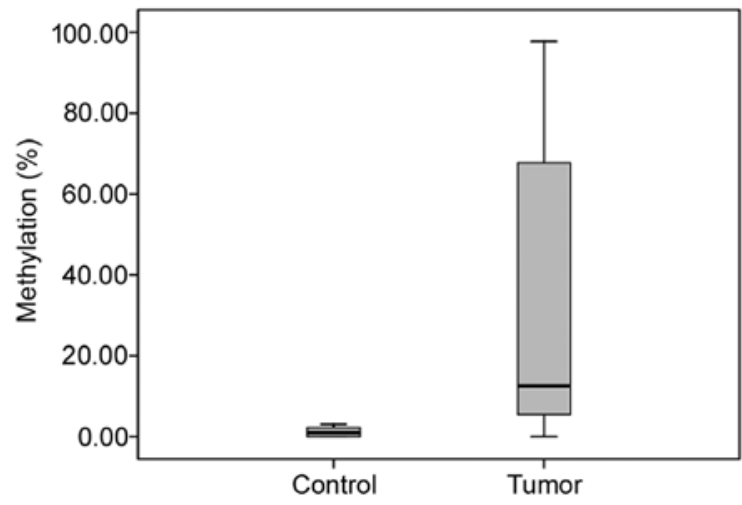

Figure 3. Comparison of promoter methylation levels of SHP1 in primary central nervous system lymphoma (PCNSL) tumor tissues and paired peripheral blood. PCNSL tumor tissues had higher methylation levels of SHP1 ( $31.7 \pm 36.5$ vs. $1.2 \pm 1.1 \%, \mathrm{p}<0.001)$. SHP1, Src homology region 2 domaincontaining phosphatase-1.

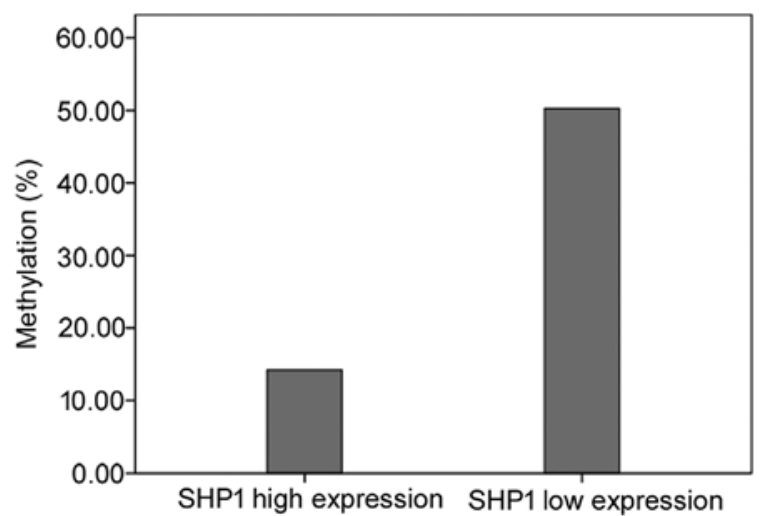

Figure 4. Comparison of promoter methylation levels of SHP1 in the SHP1 protein low expression group and high expression group. The methylation level was higher in the low expression SHP1 protein group than this level in the high expression SHP1 protein group ( $\mathrm{p}=0.004)$. SHP1, Src homology region 2 domain-containing phosphatase-1.

less than $60 \%$ of positive cells in the tissues, including 9 cases of (2+) and 4 cases of (+), respectively (Fig. 1C and D).

SHP1 protein expression is correlated with promoter methylation status in PCNSL patients. The SHP1 mean methylation level in the control samples was $1.2 \pm 1.1 \%$. SHP1 promoter methylation was detected in $29 / 33$ of the tumor tissues $(87.9 \%)$ with a mean methylation level of $31.7 \pm 36.5 \%$. In this study, PCNSL tissues with a mean methylation level of $>3.4 \%$ (mean \pm 2SD of the control samples) were considered to have a bona fide methylation state, otherwise they were considered unmethylated (Fig. 2). Compared with the methylation level of the PCNSL samples, no significant methylation was observed in the control samples ( $\mathrm{p}<0.001)$ (Fig. 3).

In 15 patients, the tumor tissues showed a low level of SHP1 expression and promoter methylation, and 3 unmethylated patients showed a high SHP1 expression. However, one unmethylated patient had a decreased SHP1 protein expression. The methylation levels in patients with a low SHP1 protein expression were higher than those with a high SHP1 protein expression $(50.3 \pm 38.9$ vs. $14.2 \pm 24.0 \%, \mathrm{p}=0.004)$ (Fig. 4).
Table II. Clinical characteristics of the patients with PCNSL in relation to SHP1 protein expression (Fisher's exact test).

\begin{tabular}{|c|c|c|c|}
\hline \multirow[b]{2}{*}{ Variables } & \multicolumn{2}{|c|}{$\begin{array}{l}\text { SHP1 protein expression } \\
\qquad(\mathrm{n}=33), \mathrm{n}(\%)\end{array}$} & \multirow[b]{2}{*}{ P-value } \\
\hline & $\begin{array}{l}\text { Low } \\
(n=16)\end{array}$ & $\begin{array}{l}\text { High } \\
(n=17)\end{array}$ & \\
\hline \multicolumn{4}{|l|}{ Age (years) } \\
\hline$>60$ & $7(58.33)$ & $5(41.67)$ & 0.48 \\
\hline$\leq 60$ & $9(42.86)$ & $12(57.14)$ & \\
\hline \multicolumn{4}{|l|}{ Gender } \\
\hline Male & $11(50.00)$ & $11(50.00)$ & 1.00 \\
\hline Female & $5(45.45)$ & $6(54.55)$ & \\
\hline \multicolumn{4}{|l|}{ LDH } \\
\hline Elevated & $10(76.92)$ & $3(23.08)$ & 0.03 \\
\hline Normal & $7(35.00)$ & $13(65.00)$ & \\
\hline \multicolumn{4}{|c|}{ Immunophenotype } \\
\hline GCB & $3(75.00)$ & $1(25.00)$ & 0.34 \\
\hline non-GCB & $13(44.83)$ & $16(55.17)$ & \\
\hline \multicolumn{4}{|c|}{ No. of lesions } \\
\hline 1 & $5(41.67)$ & $7(58.33)$ & 0.72 \\
\hline At least 2 & $11(52.38)$ & $10(47.62)$ & \\
\hline \multicolumn{4}{|c|}{$\begin{array}{l}\text { ECOG performance } \\
\text { status }\end{array}$} \\
\hline 0 to 1 & $5(50.00)$ & $5(50.00)$ & 1.00 \\
\hline At least 2 & $11(47.83)$ & $12(52.17)$ & \\
\hline \multicolumn{4}{|c|}{$\begin{array}{l}\text { Deep structure } \\
\text { involvement }\end{array}$} \\
\hline Presence & $11(45.83)$ & $13(54.17)$ & 0.71 \\
\hline Absence & $5(55.56)$ & $4(44.44)$ & \\
\hline
\end{tabular}

PCNSL, primary central nervous system lymphoma; SHP1, Src homology region 2 domain-containing phosphatase-1; LDH, lactate dehydrogenase; GCB, germinal center B-cell-like; ECOG, Eastern Cooperative Oncology Group.

Low SHP1 expression was present more frequently in PCNSL patients with elevated LDH than those with a normal LDH level (76.92 vs. $35.00 \%, p=0.03$ ) (Table II), while SHP1 protein expression was not related to the indicated clinicopathologic variables routinely detected in PCNSL.

Promoter methylation downregulates SHPI expression and phosphorylation levels in PCNSL tissues. For the samples used for western blot analysis, expression levels of SHP1, pSHP1 and STAT3 were detected and analyzed. Compared with samples with unmethylation of the SHP1 promoter and controls, decreased SHP1 and pSHP1 expression was observed in samples with SHP1 promoter methylation. Contrarily, the STAT3 expression was substantially elevated, showing that SHP1 promoter methylation was associated with a decrease in SHP1 $(p=0.001)$ and pSHP1 $(p=0.005)$, and an increase in STAT3 $(p=0.020)$ in PCNSL. The experiments were performed 


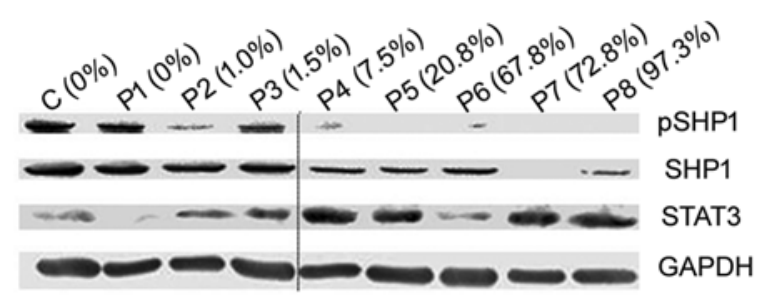

Figure 5. SHP1 expression is associated with pSHP1 and STAT3. Decreased expression of SHP1 induced downregulation of pSHP1, whereas it upregulated STAT3. C, control; P1-8, PCNSL cases; (\%), the mean methylation level of the SHP1 promoter. SHP1, Src homology region 2 domain-containing phosphatase-1.

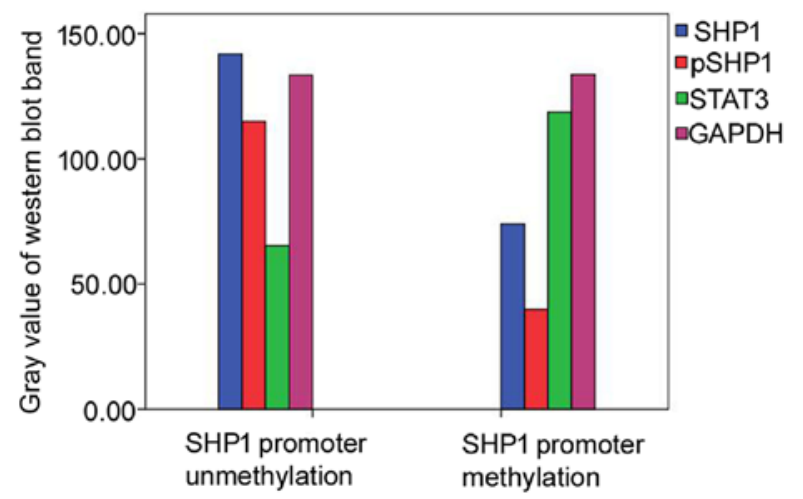

Figure 6. Comparison of gray values of the western blot bands in the methylated and unmethylated SHP1 promoter. The expression of SHP1 and pSHP1 had lower gray values in the western blot band in the methylated SHP1 promoter group compared with the unmethylated SHP1 promoter group $(\mathrm{p}=0.001$ and $\mathrm{p}=0.005)$, whereas STAT3 had higher gray values in the western blot band in the methylated SHP1 promoter group compared with the unmethylated SHP1 promoter group $(\mathrm{p}=0.020)$. SHP1, Src homology region 2 domain-containing phosphatase-1.

three times on different samples and a representative result is shown in Figs. 5 and 6.

\section{Discussion}

PCNSL is a rare form of extranodal lymphoma confined to the central nervous system. Largely due to the low incidence and obstacles to obtain tumor tissues, researchers are facing great challenges for a complete recognition of PCNSL via genomic analysis. Although the underlying genetic basis still remains unclear, existing evidence has uncovered the constitutive activation of JAK1, STAT3 and STAT6 in PCNSL. The constitutive activation of the JAK/STAT signaling pathway can induce sustained survival. Defining the key molecular pathways in PCNSL development would provide clues for targeted therapies against these malignancies.

In the present study, we confirmed that SHP1 protein was expressed in PCNSL tissues. The majority of PCNSL patients showed strong SHP1 protein expression, in line with results reported by one previous study on PCNSL (24). Sugita et al showed that SHP1 expression in PCNSL reflects the origin of PCNSL from a late germinal center to an early postgerminal center stage (24). Kossev et al showed that normal B cells in the mantle and marginal zones as well as plasma cells uniformly express SHP1. However, specific areas of the germinal centers showed no SHP1 expression (31). These results collectively suggest that SHP1 is downregulated at antigen-dependent stages of B-cell proliferation and differentiation. Moreover, this pattern of SHP1 protein expression was also observed in small B cell lymphomas, including mantle cell lymphoma, marginal zone lymphoma and chronic lymphocytic leukemia/small lymphocytic lymphoma whereas follicle center cell lymphoma did not. In this study, SHP1 expression was positive in the majority of PCNSL cases with lower expression of germinal center marker CD10, confirming their post-germinal center origin. Notably, we found that 17 of 30 SHP1-positive cases were scored as $3+$, showing a percentage less than the result reported by Sugita et al who found that all SHP1-positive cases strongly expressed and were scored as 3+ (24). By comparing the results vis-à-vis, we believe that the alteration in the SHP1 expression profile in PCNSL per se and experimental variations may contribute to the discrepancy in expression intensity interpretations.

Our results indicate that SHP1 expression and phosphorylation are suppressed by SHP1 promoter methylation. SHP1 gene methylation associated with loss of SHP1 protein has been demonstrated in many hematologic cancers. To clarify the difference in SHP1 protein expression intensity, we performed pyrosequencing analysis and western blot analysis. We found that SHP1 promoter methylation occurred in 29 of 33 PCNSL cases, and aberrant promoter SHP1 promoter methylation decreased the SHP1 protein expression, in accordance with the results noted in extranodal lymphomas $(32,33)$. SHP1 plays a critical role in the regulation of the JAK/STAT pathway $(15,34,35)$. The biological features of SHP1 were emphasized by the 'moth-eaten' (mev/mev) mouse model, which showed markedly decreased SHP1 expression and defects in myeloid cell function with a tendency for developing lymphomas $(36,37)$. SHP1 gene methylation associated with loss of SHP1 protein has been demonstrated in many hematologic cancers. In view of the normal biological significance of SHP1, SHP1 likely plays an important role in the pathogenesis of these cancers. Our data indicate that abnormal SHP1 expression contributes to the constitutive activation of the JAK/STAT signaling pathway in the pathogenesis of PCNSL.

In addition, our study demonstrated that SHP1 promoter methylation did not correlate with a complete absence of SHP1 protein expression. Several studies have also indicated this phenomenon and have suggested that monoallelic promoter methylation may not sufficiently cause complete loss of gene expression or promoter methylation, only in a small portion of tumor cells $(38,39)$, presumably due to the fact that promoter methylation may be one of the multiple causative mechanisms in tumor cells. Notably, this phenomenon was not limited to the SHP1 gene (38-41). In this study, the SHP1 promoter methylation did not cause a complete loss but a decrease in SHP1 protein expression, and the phosphorylation level reflecting the biological activities of SHP1 protein was synchronously decreased. Notably, our further analysis of the downstream signals indicated that the absence of or reduction in the phosphatase activity of SHP1 contributed to STAT3 activation in PCNSL. These results support the notion that promoter methylation contributes to decreased SHP1 protein expression and function, eventually affecting the pathogenesis of PCNSL. 
Additionally, in our study, a low-level expression of SHP1 was associated with an elevated LDH level, which was similar to previous reports $(42,43)$. Our preliminary study showed that a high LDH concentration was associated with a poor outcome in PCNSL (44). Currently, the prognostic value of SHP1 protein expression and SHP1 promoter methylation has not been well acknowledged in PCNSL. Nevertheless, in other tumor types including DLBCL, anaplastic large cell lymphoma (ALCL), prostate cancer and high-grade glioma, SHP1 has been proposed as a prognostic factor $(42,45,46)$. In these studies, the SHP1 promoter methylation status and loss of SHP1 protein expression commonly indicate a poor prognosis.

Given that PCNSL increasingly occurs in elderly populations, in which a large proportion of patients are not eligible for routine intensive therapies such as HD-MTX chemotherapy, novel therapies which target key survival molecular points in signaling pathways of PCNSL would enhance the overall patient outcomes. In view of the fact that SHP1 promoter methylation and constitutive activation of the JAK/STAT pathway are co-existent in PCNSL, epigenetic therapeutic strategies by clinically available DNA demethylation agent 5-aza-2'-deoxycytidine may be an effective approach. Most importantly, the demethylation anchor drug 5-aza-2'-deoxycytidine penetrates the bloodbrain barrier, providing further merit for the potential clinical application. Currently, how to achieve an effective and safe concentration of such agents in the central nervous system is a critical challenge. Thus, further prospective studies are needed to validate these results and to develop optimal therapies using epigenetic modifiers for PCNSL treatment.

In conclusion, we report in the present study that aberrant methylation of the SHP1 promoter is correlated with decreased SHP1 expression and phosphorylation. Attenuation of the biological functions of SHP1 contributes to the activation of transcription factor STAT3, which may affect the pathogenesis of PCNSL. Epigenetic modifiers such as 5-aza-2'-deoxycytidine may be of significance for the development of methylation-targeted therapeutic regimens.

\section{Acknowledgements}

This study was supported by grants from the National Natural Science Foundation of China (no. 81272842 awarded to Y.L. and no. 81500157 awarded to Q.C.). We thank Dr Young Whang for review of the manuscript.

\section{References}

1. Batchelor T and Loeffler JS: Primary CNS lymphoma. J Clin Oncol 24: 1281-1288, 2006.

2. Wang CC, Carnevale J and Rubenstein JL: Progress in central nervous system lymphomas. Br J Haematol 166: 311-325, 2014.

3. Campo E, Swerdlow SH, Harris NL, Pileri S, Stein H and Jaffe ES: The 2008 WHO classification of lymphoid neoplasms and beyond: Evolving concepts and practical applications. Blood 117: 5019-5032, 2011.

4. Rubenstein JL, Fridlyand J, Shen A, Aldape K, Ginzinger D, Batchelor T, Treseler P, Berger M, McDermott M, Prados M, et al: Gene expression and angiotropism in primary CNS lymphoma. Blood 107: 3716-3723, 2006.

5. Ponzoni M, Issa S, Batchelor TT and Rubenstein JL: Beyond high-dose methotrexate and brain radiotherapy: Novel targets and agents for primary CNS lymphoma. Ann Oncol 25: 316-322, 2014.
6. Jordanova ES, Riemersma SA, Philippo K, Giphart-Gassler M, Schuuring E and Kluin PM: Hemizygous deletions in the HLA region account for loss of heterozygosity in the majority of diffuse large B-cell lymphomas of the testis and the central nervous system. Genes Chromosomes Cancer 35: 38-48, 2002.

7. Tun HW, Personett D, Baskerville KA, Menke DM, Jaeckle KA Kreinest P, Edenfield B, Zubair AC, O'Neill BP, Lai WR, et al: Pathway analysis of primary central nervous system lymphoma. Blood 111: 3200-3210, 2008.

8. Booman M, Szuhai K, Rosenwald A, Hartmann E, KluinNelemans H, de Jong D, Schuuring E and Kluin P: Genomic alterations and gene expression in primary diffuse large B-cell lymphomas of immune-privileged sites: The importance of apoptosis and immunomodulatory pathways. J Pathol 216: 209-217, 2008.

9. Sung CO, Kim SC, Karnan S, Karube K, Shin HJ, Nam DH, Suh YL, Kim SH, Kim JY, Kim SJ, et al: Genomic profiling combined with gene expression profiling in primary central nervous system lymphoma. Blood 117: 1291-1300, 2011.

10. Deckert M, Montesinos-Rongen M, Brunn A and Siebert R: Systems biology of primary CNS lymphoma: From genetic aberrations to modeling in mice. Acta Neuropathol 127: 175-188, 2014.

11. Komohara Y, Horlad H, Ohnishi K, Ohta K, Makino K, Hondo H, Yamanaka R, Kajiwara K, Saito T, Kuratsu J, et al: M2 macrophage/microglial cells induce activation of Stat3 in primary central nervous system lymphoma. J Clin Exp Hematop 51: 93-99, 2011.

12. Yang SH, Lee KS, Kim IS, Hong JT, Sung JH, Son BC, Lee SW and Hong YK: Long-term survival in primary CNS lymphoma treated by high-dose methotrexate monochemotherapy: Role of STAT6 activation as prognostic determinant. J Neurooncol 92: 65-71, 2009.

13. Rubenstein JL, Wong VS, Kadoch C, Gao HX, Barajas R, Chen L, Josephson SA, Scott B, Douglas V, Maiti M, et al: CXCL13 plus interleukin 10 is highly specific for the diagnosis of CNS lymphoma. Blood 121: 4740-4748, 2013.

14. Zhu BM, Ishida Y, Robinson GW, Pacher-Zavisin M, Yoshimura A, Murphy PM and Hennighausen L: SOCS3 negatively regulates the gp130-STAT3 pathway in mouse skin wound healing. J Invest Dermatol 128: 1821-1829, 2008.

15. Nakata K, Suzuki Y, Inoue T, Ra C, Yakura H and Mizuno K: Deficiency of SHP1 leads to sustained and increased ERK activation in mast cells, thereby inhibiting IL-3-dependent proliferation and cell death. Mol Immunol 48: 472-480, 2011.

16. Yi TL, Cleveland JL and Ihle JN: Protein tyrosine phosphatase containing SH2 domains: Characterization, preferential expression in hematopoietic cells, and localization to human chromosome 12p12-p13. Mol Cell Biol 12: 836-846, 1992.

17. Dixon JE: Protein tyrosine phosphatases: their roles in signal transduction. Recent Prog Horm Res 51: 405-415, 1996.

18. Healy JI and Goodnow CC: Positive versus negative signaling by lymphocyte antigen receptors. Annu Rev Immunol 16: 645-670, 1998.

19. Wu C, Sun M, Liu L and Zhou GW: The function of the protein tyrosine phosphatase SHP-1 in cancer. Gene 306: 1-12, 2003.

20. Chim CS, Wong AS and Kwong YL: Epigenetic dysregulation of the Jak/STAT pathway by frequent aberrant methylation of SHP1 but not SOCS1 in acute leukaemias. Ann Hematol 83: 527-532, 2004.

21. Chen HE, Chang S, Trub $\mathrm{T}$ and Neel BG: Regulation of colony-stimulating factor 1 receptor signaling by the $\mathrm{SH} 2$ domain-containing tyrosine phosphatase SHPTP1. Mol Cell Biol 16: 3685-3697, 1996.

22. Law CL, Sidorenko SP, Chandran KA, Zhao Z, Shen SH, Fischer EH and Clark EA: CD22 associates with protein tyrosine phosphatase 1C, Syk, and phospholipase C-gamma(1) upon B cell activation. J Exp Med 183: 547-560, 1996.

23. Yi T, Zhang J, Miura O and Ihle JN: Hematopoietic cell phosphatase associates with erythropoietin (Epo) receptor after Epo-induced receptor tyrosine phosphorylation: Identification of potential binding sites. Blood 85: 87-95, 1995.

24. Sugita Y, Tokunaga O, Nakashima A and Shigemori M: SHP-1 expression in primary central nervous system B-cell lymphomas in immunocompetent patients reflects maturation stage of normal B cell counterparts. Pathol Int 54: 659-666, 2004.

25. Oka T, Ouchida M, Koyama M, Ogama Y, Takada S, Nakatani Y, Tanaka T, Yoshino T, Hayashi K, Ohara N, et al: Gene silencing of the tyrosine phosphatase SHP1 gene by aberrant methylation in leukemias/lymphomas. Cancer Res 62: 6390-6394, 2002. 
26. Harris NL, Jaffe ES, Stein H, Banks PM, Chan JK, Cleary ML, Delsol G, De Wolf-Peeters C, Falini B, Gatter KC, et al: A revised European-American classification of lymphoid neoplasms: A proposal from the International Lymphoma Study Group. Blood 84: 1361-1392, 1994.

27. Patel B, Chacko G, Nair S, Anandan J, Chacko AG, Rajshekhar V and Turel M: Clinicopathological correlates of primary central nervous system lymphoma: Experience from a tertiary care center in South India. Neurol India 63: 77-82, 2015.

28. Mounier N, Briere J, Gisselbrecht C, Emile JF, Lederlin P, Sebban C, Berger F, Bosly A, Morel P, Tilly H, et al: Rituximab plus CHOP (R-CHOP) overcomes bcl-2--associated resistance to chemotherapy in elderly patients with diffuse large B-cell lymphoma (DLBCL). Blood 101: 4279-4284, 2003.

29. Feng W, Shen L, Wen S, Rosen DG, Jelinek J, Hu X, Huan S Huang M, Liu J, Sahin AA, et al: Correlation between CpG methylation profiles and hormone receptor status in breast cancers. Breast Cancer Res 9: R57, 2007.

30. Hans CP, Weisenburger DD, Greiner TC, Gascoyne RD, Delabie J, Ott G, Müller-Hermelink HK, Campo E, Braziel RM, Jaffe ES, et al: Confirmation of the molecular classification of diffuse large B-cell lymphoma by immunohistochemistry using a tissue microarray. Blood 103: 275-282, 2004.

31. Kossev PM, Raghunath PN, Bagg A, Schuster S, Tomaszewski JE and Wasik MA: SHP-1 expression by malignant small B-cell lymphomas reflects the maturation stage of their normal B-cell counterparts. Am J Surg Pathol 25: 949-955, 2001.

32. Koyama M, Oka T, Ouchida M, Nakatani Y, Nishiuchi R, Yoshino T, Hayashi K, Akagi T and Seino Y: Activated proliferation of B-cell lymphomas/leukemias with the SHP1 gene silencing by aberrant CpG methylation. Lab Invest 83: 1849-1858, 2003.

33. Han Y, Amin HM, Frantz C, Franko B, Lee J, Lin Q and Lai R: Restoration of shp1 expression by 5-AZA-2'-deoxycytidine is associated with downregulation of JAK3/STAT3 signaling in ALK-positive anaplastic large cell lymphoma. Leukemia 20: 1602-1609, 2006.

34. Bittorf T, Seiler J, Zhang Z, Jaster R and Brock J: SHP1 protein tyrosine phosphatase negatively modulates erythroid differentiation and suppression of apoptosis in J2E erythroleukemic cells. Biol Chem 380: 1201-1209, 1999.

35. Jiao H, Berrada K, Yang W, Tabrizi M, Platanias LC and Yi T: Direct association with and dephosphorylation of Jak2 kinase by the $\mathrm{SH} 2$-domain-containing protein tyrosine phosphatase SHP-1. Mol Cell Biol 16: 6985-6992, 1996.

36. Kozlowski M, Mlinaric-Rascan I, Feng GS, Shen R, Pawson T and Siminovitch KA: Expression and catalytic activity of the tyrosine phosphatase PTP1C is severely impaired in motheaten and viable motheaten mice. J Exp Med 178: 2157-2163, 1993.
37. Ma XZ, Jin T, Sakac D, Fahim S, Zhang X, Katsman Y, Bali M and Branch DR: Abnormal splicing of SHP-1 protein tyrosine phosphatase in human $\mathrm{T}$ cells. Implications for lymphomagenesis. Exp Hematol 31: 131-142, 2003.

38. Brell M, Tortosa A, Verger E, Gil JM, Viñolas N, Villá S, Acebes JJ, Caral L, Pujol T, Ferrer I, et al: Prognostic significance of $O^{6}$-methylguanine-DNA methyltransferase determined by promoter hypermethylation and immunohistochemical expression in anaplastic gliomas. Clin Cancer Res 11: 5167-5174, 2005.

39. Baeza N, Weller M, Yonekawa Y, Kleihues P and Ohgaki H: PTEN methylation and expression in glioblastomas. Acta Neuropathol 106: 479-485, 2003.

40. Cameron EE, Baylin SB and Herman JG: p15(INK4B) CpG island methylation in primary acute leukemia is heterogeneous and suggests density as a critical factor for transcriptional silencing. Blood 94: 2445-2451, 1999.

41. Lenz G, Hutter G, Hiddemann W and Dreyling M: Promoter methylation and expression of DNA repair genes hMLH1 and MGMT in acute myeloid leukemia. Ann Hematol 83: 628-633, 2004.

42. Amara K, Trimeche M, Ziadi S, Laatiri A, Hachana M and Korbi S: Prognostic significance of aberrant promoter hypermethylation of $\mathrm{CpG}$ islands in patients with diffuse large B-cell lymphomas. Ann Oncol 19: 1774-1786, 2008

43. Khoury JD, Rassidakis GZ, Medeiros LJ, Amin HM and Lai R: Methylation of SHP1 gene and loss of SHP1 protein expression are frequent in systemic anaplastic large cell lymphoma. Blood 104: 1580-1581, 2004

44. Liu J, Sun XF, Qian J, Bai XY, Zhu H, Cui QU, Li XY, Chen YD, Wang YM and Liu YB: Immunochemotherapy for primary central nervous system lymphoma with rituximab, methotrexate, cytarabine and dexamethasone: Retrospective analysis of 18 cases. Mol Clin Oncol 3: 949-953, 2015.

45. Tassidis H, Brokken LJ, Jirström K, Ehrnström R, Pontén F, Ulmert D, Bjartell A, Härkönen P and Wingren AG: Immunohistochemical detection of tyrosine phosphatase SHP-1 predicts outcome after radical prostatectomy for localized prostate cancer. Int J Cancer 126: 2296-2307, 2010.

46. Zhang L, Wang M, Wang W and Mo J: Incidence and prognostic value of multiple gene promoter methylations in gliomas. J Neurooncol 116: 349-356, 2014. 